\title{
Cytopenias in Transplant Patients
}

\author{
Maura Barry, Sunandana Chandra, and Kenneth B. Hymes
}

\section{Anemia in Solid Organ Transplants}

Anemia is commonly seen after solid organ transplant (SOT). It can result from a number of different etiologies including bleeding or hemorrhage, iron deficiency, hemolysis, drug related, lack of erythropoietin, marrow suppression, and congenital etiologies. The frequency and severity of anemia is dependent on the type of solid organ transplant and the duration of time that has passed since the transplant. Much of the data for the causes of anemia following SOT is from renal transplant and cardiac transplant populations. In these renal transplant patients, the prevalence of anemia is about $40 \% 1$-year posttransplant. Severe anemia, defined as $\mathrm{Hb}<11 \mathrm{~g} / \mathrm{dL}$ in males and $10 \mathrm{~g} / \mathrm{dL}$ in females, is seen in $8.5 \%$ of patients $6-60$ months post-kidney transplant [1]. Anemia to a hematocrit of less than $33 \%$ can persist for greater than 5 years in $25 \%$ patients and can result in worsened renal graft function and graft loss $[2,3]$. Pre-transplant anemia often seen in dialysis can often improve posttransplant, but then may recur if there is graft failure. About $40 \%$ of cardiac transplant patients also experience posttransplant anemia [4]. Pre-transplant anemia that is seen in $25 \%$ of this population negatively affects 1-year survival posttransplant [5].

\section{Barry $(\square)$}

Division of Hematology and Oncology, Larner College of

Medicine at the University of Vermont, University of Vermont

Medical Center, Burlington, VT, USA

e-mail: maura.barry@uvmhealth.org

\section{S. Chandra}

Division of Hematology and Oncology, Northwestern University

Feinberg School of Medicine, Chicago, IL, USA

e-mail: sunandana.chandra@northwestern.edu

\section{K. B. Hymes}

Hematology, Coagulation, and Medical Oncology, Laura and Isaac Perlmutter Cancer Center, NYU Langone Health,

New York, NY, USA

\section{Hemolysis}

Mismatched ABO solid organ transplantation is often employed due to the shortage of transplantable organs. Three different groups of ABO incompatibility can be found in transplantation: minor, major, and bidirectional. Complications arising from minor ABO-mismatched solid organ transplants include the passenger lymphocyte syndrome (PLS) [6]. Recipients of minor ABO-incompatible transplantation express $\mathrm{ABO}$ antigens that are not expressed in the donor and may result in a graft-versus-host $(\mathrm{GvH})$ reaction, including delayed hemolysis of recipient red blood cells [7]. Passenger lymphocyte syndrome occurs when antibodies that are produced by the donor B-lymphocytes result in a primary or secondary immune response against the recipient's $\mathrm{ABO}$ and $\mathrm{Rh}$ antigens. The severity of hemolysis depends on the level of red cell isoagglutinins in the donor tissue that are passively transferred with the organ and the subsequent rise in antibodies in the transplant recipient that occurs 1-3 weeks posttransplant and usually resolves within 3 months posttransplant [7]. In rare instances, PLS can occur due to non- $\mathrm{ABO} / \mathrm{Rh}$ antibodies if the organ had been previously sensitized to other red cell antigens in the setting of pregnancy or transfusion [8-11]. PLS occurs more frequently in the heart and lung transplants and less frequently in liver and kidney transplants [7].

\section{Drugs}

There are numerous drugs that are often used in the solid organ transplant setting that can cause myelosuppression, including anemia, through a variety of pathophysiologic mechanisms. A number of immunosuppressants with various pharmacologic mechanisms of action are used to prolong graft and recipient survival. The immunosuppressants mycophenolate mofetil and tacrolimus have been shown to cause anemia in renal transplant recipients [12]. One-year posttransplant, renal transplant patients with anemia who are on 
mycophenolate mofetil have a lower rates of survival and higher rates of cardiovascular death [13]. Sirolimus, another immunosuppressant, may result in greater myelosuppression compared to mycophenolate mofetil [14]. Sirolimus and calcineurin inhibitors such as tacrolimus and cyclosporine have been shown in renal and lung transplant recipients to cause hemolytic anemia, thrombotic thrombocytopenic purpura, and atypical hemolytic uremic syndrome [15-17]. The calcineurin inhibitors have been shown to cause anemia ranging from $1 \%$ to $5 \%$ in European trials to $38-47 \%$ in US trial [18]. The antimetabolite azathioprine, a purine-analog drug, can also cause cytopenias. Mycophenolate mofetil, tacrolimus, azathioprine, and anti-thymocyte globulin have all shown to cause pure red cell aplasia [19, 20].

Primaquine and dapsone are used for PCP treatment, and both can result in hemolysis in glucose-6-phosphatedehydrogenase-deficient patients, which is not restricted to solid organ transplant settings. In patients with low body weight or renal failure, dapsone may induce a hemolytic anemia and produce methemoglobinemia even if the G6PD levels are normal [21-23].

Ribavirin and interferon can cause bone marrow suppression in liver transplant recipients who are being treated with recurrent hepatitis C virus [24]. Ribavirin is used in treating respiratory syncytial virus after transplant in both the oral and inhaled formulations, both of which can cause bone marrow suppression [25]. In patients who are co-infected with human deficiency virus (HIV) and hepatitis $\mathrm{C}$ virus, myelosuppression can be seen with the anti-retroviral medication, AZT, and the anemia can be exacerbated with the coadministration of ribavirin. The antibiotic trimethoprimsulfamethoxazole can also cause myelosuppression including anemia. Valganciclovir has been reported to cause bone marrow failure in renal transplant patients who received this antiviral as prophylaxis [26].

Newer immunosuppressants have been developed which allow for the sparing of steroids and calcineurin inhibitors, the latter of which can cause chronic nephropathy. These newer agents include alemtuzumab, a human anti-CD52 antibody that depletes T- and B-cells, daclizumab, a human anti-CD25 antibody that targets the IL-2 alpha subunit, and anti-thymocyte globulin (ATG). Alemtuzumab has been reported to be associated with red cell aplasia, autoimmune hemolytic anemia, and idiopathic thrombocytopenia purpura in pancreas transplant patients [27].

\section{Iron Deficiency}

Iron deficiency is often overlooked in transplant patients. In renal transplant patients, those with a hematocrit of less than $30 \%$ have iron studies checked only $40 \%$ of the time [28]. Perioperative bleeding and frequent phlebotomies for labo- ratory studies can contribute to iron deficiency anemia. Anemia of chronic disease is also frequently seen in the transplant population due to chronic inflammation, abnormal erythropoietin production due to allograft nephropathy after renal transplant. Drugs such as ACE inhibitors that are often used in chronic kidney disease are also associated postkidney transplant anemia [1].

\section{Infections}

Numerous infectious etiologies that can occur during the posttransplant immunosuppressed period have been shown to cause myelosuppression including anemia. Parvovirus B19, a single-stranded DNA virus, has been known to cause red cell aplasia with anemia, reticulocytopenia, and erythroid maturation arrest [29]. Elevated parvoviral B19 titers have been found by PCR in lung transplant recipients who had anemia with other etiologies that were ruled out [30-32]. Cytomegalovirus (CMV) infection as well as its first-line therapies, ganciclovir and valganciclovir, can be associated with bone marrow suppression. Also tuberculosis, histoplasmosis, Epstein-Barr virus (EBV), human herpes virus- 6 , and human herpes virus- 8 infections can be associated with bone marrow suppression and pancytopenia [33]. Posttransplant lymphoproliferative disorder that can be seen with immunosuppressive therapy can also be associated with pancytopenia.

\section{Posttransplant Lymphoproliferative Disorder}

Posttransplant lymphoproliferative disorder (PTLD) which includes the spectrum of infectious mononucleosis, EBVdriven polyclonal lymphocyte proliferation, and nonHodgkin's lymphoma can be seen with solid organ transplantation [29]. PTLD is due to the impaired EBVspecific cytotoxic T-cell activity that allows for recipient $B$ cells that have latent EBV infection to expand. PTLD can result in bone marrow infiltration and pancytopenia, as well as cause autoimmune hemolytic anemia. The severity of PTLD depends on the level of immunosuppression and usually occurs within the 1 st year after transplant.

\section{Graft-Versus-Host Disease}

Graft-versus-host disease (GVHD) is rarely seen after SOT and is due to the engraftment and proliferation of allograftassociated lymphocytes in the immunosuppressed transplant recipient causing an immune-mediated response toward HLA-unmatched host tissue. Risk factors for the development of GVHD includes the volume of lymphoid 
tissue that is transplanted and therefore is seen more with small bowel and liver transplants, in those over 65 years of age and with HLA mismatch between donor and recipient $[34,35]$. GVHD, in contrast to the development of PTLD, occurs early after SOT, on the order of weeks to months depending on the type of solid organ transplant. The clinical presentation usually includes fever, rash, diarrhea, and cytopenias, and diagnosis is made by histologic confirmation of affected tissue.

\section{Hemophagocytic Syndrome}

Hemophagocytic syndrome is a systemic inflammatory disease that can include the symptoms of fever, hepatosplenomegaly, lymphadenopathy, pancytopenia, rash, jaundice, cough, dyspnea, cachexia, and neurologic dysfunction and can often occur in response to a precipitant, such as infection [29]. This syndrome is a result of aberrant immune response of abnormal T-cell activation leading to hemophagocytosis by activated, nonmalignant macrophages that secrete numerous cytokines including interleukin (IL)-1, IL-6, IL-12, and tumor necrosis factor-alpha in the bone marrow, liver, lymph nodes, and spleen, resulting in a "cytokine storm" [29]. Acquired hemophagocytic syndrome has been documented in the renal, liver, heart, and pancreaskidney solid organ transplants. There have been cases of hemophagocytic syndrome due to disseminated histoplasmosis in renal transplant recipients which were diagnosed by bone marrow biopsy [36].

\section{Leukopenia in Solid Organ Transplants}

Leukopenia can be defined as having a white blood cell (WBC) count of less than 3000-4000 cells/ $\mu \mathrm{L}$, with neutropenia defined as an absolute neutrophil count (ANC) <500/ $\mathrm{mm}^{3}$ by the Infectious Diseases Society of America [37]. Leukopenia is commonly seen after solid organ transplantation and can be caused by noninfectious and infectious etiologies. It can signal an underlying infection or disease process, such as posttransplant lymphoproliferative disorder (PTLD). It also increases the risk of developing further complications such as opportunistic infection and can require reduction of immunosuppression, increasing the risk of graft rejection. While there is no data to suggest a clear independent relationship between leukopenia and graft rejection, the complications of leukopenia mentioned above provide ample reason to investigate the etiology of the decreased white cell count. Solid organ transplant recipients are at risk for developing infections due to their medically induced immunodeficiency following transplant, required to prevent rejection of the transplanted organ.

\section{Noninfectious Etiologies}

Noninfectious causes of leukopenia include drugs that are often used in transplant settings. Numerous immunosuppressants can cause leukopenia, but given their use in combination, it is difficult to elucidate each agent's individual role in incidence and management. In one retrospective study of adult kidney and pancreas transplantations, the incidence of either leukopenia or neutropenia was $58 \%$, with the first episode occurring at a mean of 91 days posttransplant [38].

One of the most common immunosuppressants, azathioprine, is a purine analog that causes an antimetabolite effect. Azathioprine may result in leukopenia in a dose-dependent manner, as well as based on the duration of treatment. The leukopenia that results from azathioprine is usually reversible upon dose-reduction or drug discontinuation. The leukopenia, often occurring late in the course of therapy, can be related to low or absent levels of S-methyl-transferase (TPMT) activity, which metabolizes 6-mercaptopurine, and can result in increased myelotoxicity [39].

Drugs that result in the depletion of T cells, such as thymoglobulin and alemtuzumab, can also lead to leukopenia in $10-14 \%$ of patients [40]. The immunosuppressant, mycophenolate mofetil (MMF), reversibly and noncompetitively inhibits the enzyme, inosine monophosphate dehydrogenase, the rate-limiting enzyme for de novo purine synthesis during lymphocyte proliferation [29]. MMF can result in leukopenia in $13-35 \%$ of patients. The myelosuppression of MMF is dose-dependent and is related to the trough level of the active metabolite, mycophenolic acid; however, brief discontinuations of the drug can lead to organ rejection, especially in the era of steroid-sparing regimens [41, 42]. The calcineurin inhibitors such as cyclosporine, tacrolimus, and sirolimus can also lead to cytopenias, including leukopenia.

\section{Infections}

Some of these agents can cause leukopenia as one of many symptoms of infection. For example, leukopenia (and often thrombocytopenia as well) have been observed as a sign/ symptoms of infection with pathogens such as adenovirus, coronavirus, lymphocytic choriomeningitis virus (LCMV), parainfluenza, ehrlichiosis, and measles [43-45]. In areas endemic for the disease, dengue infection also causes both leukopenia and thrombocytopenia in patients after solid organ transplant [46]. Fungal infections such as histoplasmosis can cause disseminated organ infiltration, with the bone marrow being a common area of involvement, resulting in decreased hematopoiesis and cytopenias [33]. Parvovirus B19, much better known for its role in causing both acute and chronic anemia in solid organ transplants, is also reported to cause acute and chronic leukopenia in approximately $37.5 \%$ of solid 
organ as well as hematopoietic stem cell transplant recipients who develop the infection [32, 47]. An acute infection with HHV-8 can present with fever, splenomegaly, and leukopenia (as part of a pancytopenia), with bone marrow biopsy revealing hypocellularity, plasma cell infiltration, and evidence of viral infection by immunohistochemical staining [33].

A retrospective analysis of liver and kidney transplant recipients was performed to assess the relationship between leukopenia and positive hepatitis $\mathrm{B}$ and $\mathrm{C}$ serologies. The investigators found that there was no significant correlation between leukopenia and hepatitis $\mathrm{C}$ infection, but that the incidence of leukopenia in those with active hepatitis $\mathrm{B}$ infection was $7.4 \%$. They posited that, similar to other viruses, infection with hepatitis B virus could lead to "decreased or ineffective leukocyte production in the bone marrow...shifts of cells from the circulation to the marginal blood pools... [and] also produce peripheral destruction of white blood cells due to immune and nonimmune processes" [48].

Cytomegalovirus infection is the most well-known transplant-related infection to cause cytopenias, with leukopenia found in approximately $20 \%$ of infected transplant recipients and with most of the data and research conducted in kidney transplant populations [49]. Infection with CMV has direct effects on the bone marrow, inhibiting hematopoiesis by affecting both the bone marrow stroma and the stem cells and hematopoietic precursors [33, 50]. CMV disease (acute symptomatic infection) is most often seen in the first 6 months, particularly during the first 3 months posttransplant, and presents with constitutional complaints such as fever, abdominal pain, diarrhea, and respiratory symptoms along with cytopenias [33, 49, 51]. However, in heart transplant patients, a subclinical infection during the 1st year where infected individuals are asymptomatic has also been associated with leukopenia, with the most significant reductions occurring in the neutrophil and monocyte populations and preservation of the lymphocyte counts [52].

An added challenge when addressing CMV infection and leukopenia results from the frequent finding that the treatments for the disease can result in further leukopenia (discussed in "Noninfectious Etiologies of Leukopenia" section).

Additional diagnoses to consider when assessing the etiologies of leukopenia, as well as pancytopenia, with regard to infection are hemophagocytic syndrome (HPS) which is associated with CMV, EBV, HHV-6, HHV-8, and histoplasmosis, as well as EBV-associated PTLD [29, 33].

\section{Thrombocytopenia and Solid Organ Transplant}

As in all cases of thrombocytopenia, when evaluating a finding of low platelets in a patient after SOT, it must be determined whether the primary problem is one of impaired production in the bone marrow or if it is a matter of consumption or sequestration outside the marrow. Infections and drugs are known to suppress megakaryocyte production in the marrow, such as cytomegalovirus and trimethoprimsulfamethoxazole (TMP-SMX). Additionally, both infections and medications as well as auto- and alloimmune processes can lead to destruction of platelets despite adequate production of megakaryocytes in the bone marrow.

\section{Infectious Etiologies of Thrombocytopenia}

Solid organ transplant recipients are at risk for developing infections due to their immunosuppression, and viral infections in particular are a potential contributor to the development of thrombocytopenia following solid organ transplant. Detailed discussions of these infections are found in other chapters of this book, but their involvement in thrombocytopenia is discussed below. In addition to the viral infections that contribute to thrombocytopenia, it is important to remember that thrombocytopenia can be a sign of bacterial infection and sepsis most often in the context of disseminated intravascular coagulation (DIC). Appropriate workup to rule out infection is among the first steps in examining thrombocytopenia in a solid organ recipient.

\section{Cytomegalovirus}

The virus of particular concern in regard to platelet count in transplant patients specifically is cytomegalovirus, though thrombocytopenia due to other viruses has also been described, often among a constellation of systemic symptoms.

Cytomegalovirus can cause thrombocytopenia both by decreasing production of and through destruction of platelets. Studies have shown that CMV can impair megakaryocyte production in its early stages by infection of stromal cells, which interferes with growth factor production, as well as by directly infecting myeloid cells [50], similar to CMVrelated leukopenia.

The other reported etiology of thrombocytopenia from CMV is due to intravascular destruction of platelets by CMV-associated thrombotic microangiopathy (TMA), with a clinical picture resembling that of thrombotic thrombocytopenic purpura (TTP)/atypical hemolytic uremic syndrome (aHUS), consisting of varying degrees of Coombs-negative hemolytic anemia, thrombocytopenia, acute kidney injury, fever, and neurological findings. While this etiology is more often identified as a drug-related phenomenon, particularly due to the immunosuppressants required to prevent organ rejection (see next section), there have been multiple case reports associating CMV infection as a trigger of TMA in the posttransplant setting $[53,54]$. This has been noted with particular frequency in the renal transplant literature, where 
both de novo and recurrent forms of aHUS were associated with CMV infection in renal transplant recipients. However, particularly in the patients with "de novo" disease, it is possible that CMV may be directly driving the thrombotic microangiopathy, rather than solely by the complementmediated events of aHUS. Mechanisms thought to be underlying CMV's endothelial effects include activation of CMV-specific cytotoxic immune responses and induction of primitive endothelial dysfunction as well as direct infection of endothelial cells by CMV $[55,56]$.

However, some investigators question how significant a contributor CMV actually is to thrombotic microangiopathy in transplant patients. In a review of TMA among lung transplant recipients by Hachem and colleagues, an analysis of 24 patients who were diagnosed with TMA following lung transplantation revealed that only 4 patients had evidence of CMV infection, and additionally that there were 229 incidences of CMV viremia among the 237 lung transplant patients who did not develop TMA. Additionally, in their univariate and multivariate analyses, neither CMV viremia nor serologic status was identified as a risk factor for TMA in the study population [57].

\section{Epstein-Barr Virus}

Infection with Epstein-Barr virus often results in conditions associated with pancytopenia, such as PTLD and hemophagocytic syndrome, both of which are described in greater detail in previous sections of this chapter (see "Leukopenia and Anemia" sections). EBV should be considered as a possible infectious etiology during the workup of thrombocytopenia, particularly if other systemic signs or symptoms are present.

\section{Other Infectious Etiologies}

Other infectious etiologies that have thrombocytopenia among the constellation of presenting symptoms that have been described in organ transplant recipients include coronavirus, particularly SARS, lymphocytic choriomeningitis virus (LCMV), and HHV-6, though the thrombocytopenia is unlikely to be the primary issue at presentation [58]. Parvovirus B-19 and polyoma BK virus infection have also been associated with development of aHUS [55, 59].

It is also important to note that chronic infection with hepatitis $\mathrm{C}$ can be an etiology for thrombocytopenia both in and outside the context of solid organ transplantation. The etiology of thrombocytopenia in the setting of hepatitis $\mathrm{C}$ infection can be due to hepatocellular damage including fibrosis and/or cirrhosis affecting thrombopoietin (TPO) production, hypersplenism due to portal hypertension, bone marrow suppression, immune dysfunction, and development of platelet autoantibodies [60]. Additionally, treatment for hepatitis $\mathrm{C}$ with interferon is known to cause thrombocytopenia.

\section{Noninfectious Etiologies of Thrombocytopenia}

There are numerous noninfectious etiologies of thrombocytopenia that have been identified in SOT patients. Many pharmacologic agents have been implicated in the development of thrombocytopenia following SOT through varying mechanisms, such as TMA, decreased megakaryocyte production, and auto- and allo-immune mechanisms of platelet destruction.

\section{Pharmacologic Agents}

\section{Calcineurin Inhibitors}

The drugs most strongly associated with decreased platelet counts due to thrombotic microangiopathy are the calcineurin inhibitors (CI) cyclosporine and tacrolimus. Calcineurin inhibitor induced TMA often occurs within weeks following SOT, and the CIs are thought to cause direct endothelial injury and platelet aggregation, although the specific mechanism has not been identified. When this is identified, numerous case studies in multiple different organ systems (lung, liver, kidney solid organ transplant) have reported that changing from one $\mathrm{CI}$ to another (tacrolimus to cyclosporine or vice versa) or to another class of medication such as sirolimus or mycophenolate mofetil can prevent further episodes of TMA from occurring [61-64]. However, the addition of the mTOR inhibitor sirolimus to a calcineurin inhibitor also increases the chance of developing TMA $[57,65]$.

\section{Antivirals and Antibiotics}

Ganciclovir and valganciclovir are used in prophylaxis and treatment of CMV. Both are known to have myelosuppressive effects, particularly on granulocytes and platelets, but generally there is rapid recovery of counts following withdrawal of the medication.

One of linezolid's most well-known adverse effects is thrombocytopenia, with the package insert reporting a rate of $3 \%$ in adults. Other studies have reported rates of grades III-IV thrombocytopenia of approximately 5.2\% [66]. No mechanism has been identified for linezolid-related thrombocytopenia, though some evidence suggests that it is an immune-mediated phenomenon [67]. The medication is frequently used in the treatment of vancomycin-resistant enterococcus (VRE), which has been an infection seen in transplant patients, as well as non-transplant patients, with increasing frequency. A multicenter compassionate use trial published in 2003 showed that it was an effective drug in treating VRE, which was identified as having a mortality rate of up to $83 \%$, with the authors reporting a $62 \%$ survival rate after treatment with linezolid. Thrombocytopenia was the main adverse effect of treatment, seen in $4.7 \%$, but did not necessitate the cessation of therapy [68]. A second study in liver transplant patients treated with linezolid for VRE infec- 
tion showed a similar treatment efficacy and again reported no cases (0/46 patients) requiring cessation of therapy due to severe thrombocytopenia, and furthermore found no correlation between treatment duration and platelet counts [69] though other articles advise caution when using linezolid for extended time periods [70]. Thus, while it may or may not require any intervention or change in treatment plan, it should be considered as part of the differential diagnosis when assessing thrombocytopenia.

\section{Heparin}

Heparin-induced thrombocytopenia is an additional drugrelated event that can occur in the setting of solid organ transplant. Assessment of this as a possible etiology for thrombocytopenia follows the same algorithm as it would for any patient receiving heparin. The probability of the thrombocytopenia being related to heparin use would be based on the 4Ts whether the timing ( $>10$ days following start of heparin use or sooner if heparin was used previously), degree of fall ( $>50 \%$ decrease from baseline), presence of thrombosis, and lack of alternate explanations for the thrombocytopenia suggest that heparin could be the causative agent [71]. Studies reveal that HIT is an uncommon occurrence in liver transplant recipients, and that thrombotic events and HIT antibody positivity were not well correlated [72, 73]. Case studies in renal transplant patients have reported some incidences of HIT posttransplant and graft-failure related to HIT, in part related to previous exposure to heparin in hemodialysis [74, 75].

HIT antibody immunoassays are often sent if a patient develops thrombocytopenia and has received heparin at any time during the hospitalization. However, the high sensitivity but low specificity of the test results in overdiagnosis of heparin-induced thrombocytopenia exposes patients to unnecessary risks associated with therapeutic anticoagulation. Chaturvedi and colleagues examined this phenomenon at Cleveland Clinic and found that utilizing the 4Ts algorithm to first rule out patients at low risk for HIT was a safe, reliable, and cost-effective [76]. Therefore, we recommend that immunoassays for HIT antibodies be utilized only in those patients whose $4 \mathrm{~T}$ scores suggest intermediate or high probability of heparin-induced thrombocytopenia.

If a HIT antibody immunoassay is sent once a patient is determined to be of intermediate/high risk for HIT, it is important to understand how this test is interpreted. The immunoassay detects the presence of antiplatelet factor 4 (PF4) antibodies in patient serum and is interpreted by optical density (OD). A higher reported OD correlates to a higher titer of the antibody and is more strongly suggestive of a diagnosis of HIT. As mentioned previously, ELISAs for HIT have a high sensitivity (meaning a negative test can rule out the diagnosis) but a low specificity, underscoring the need to first confirm a high pretest probability.

\section{Immune Etiologies}

\section{Immune Thrombocytopenic Purpura}

Immune thrombocytopenic purpura (ITP) is characterized by very low platelet counts, petechiae and bruising, as well as mucosal bleeding, due to opsonization of platelets in the circulation. Occurrence of ITP following solid organ transplant has been documented particularly in the liver transplant literature, with the cases attributed to either autoimmune ITP, at times precipitated by an identified infectious etiology such as tuberculosis [77] or alloimmune etiologies. The literature reports that chronic renal disease and renal transplant in patients with ITP are noted to be very rare [78], and thus most of our knowledge of ITP as an etiology following transplant is from the liver transplant literature.

One study reported a case series of eight patients who developed ITP following orthotopic liver transplantation (OLT), with a mean time of presentation of ITP since OLT of 5.4 years [79]. These cases were all felt to be autoimmune cases, as there was no history of ITP in the donors. This case series also presented a review of the previous literature on ITP after OLT, and they noted two distinct time patterns of ITP presentation, early (within 3 months) or late ( $>3$ months). The authors note that it has been proposed that the earlyonset presentation may be due to passive transfer of antibodies from the donor to the recipient. Those that developed late-onset ITP were felt to have developed the antibodies independently of their donors [79].

Additionally, studies have reported on development of alloimmune thrombocytopenic purpura, with antibodies introduced from donors with a history of ITP [79, 80]. One case study described a case where a donor liver was obtained from a donor who had died after a cerebral hemorrhage secondary to ITP. The recipient developed ITP within 3 days of transplant and subsequently expired after developing portal vein thrombosis. The authors attributed the death to ITP in that they were unable to anticoagulate but were providing blood products that may have resulted in increased likelihood of thrombosis. It is also possible, however, that the donor was producing procoagulant antibodies, as approximately 20-25\% of patients with ITP also have antiphospholipid antibodies [81, 82]. Based on this event, the authors recommended excluding cadaveric transplants from donors whose death is attributed to ITP [80].

\section{Other Etiologies}

Particularly in liver transplant patients, thrombocytopenia is often seen prior to transplant, and generally approximately $50 \%$ of transplant recipients develop worsening thrombocytopenia within 2 weeks following transplant. This acute decrease generally resolves within the 1 st month after trans- 
plant, and if thrombocytopenia persists, another etiology should be sought $[79,83,84]$. Thrombocytopenia following liver transplant can also be attributed to residual portal hypertension or hypersplenism, if either of these conditions persist following transplant. However, it is important to remain vigilant to other causes particularly drug-related and infectious etiologies that could cause a drop in the platelet count.

Additionally, while case reports exist of TMA with low ADAMTS13 levels attributed to inhibitors present in the blood [85], this is not a common phenomenon, and etiologies of TMA mentioned previously (infectious and drug-related) would be much more likely.

\section{Treatment}

In most cases, treatment of the underlying etiology of the thrombocytopenia will result in improvement in platelet counts. That may require antivirals, adjustment of the immunosuppressant regimen, withdrawal of other pharmacologic agents such as heparin, or supportive care. Platelet transfusions may be necessary if bleeding events occur or if additional procedures are necessary, but we do not recommend prophylactic transfusions for maintenance of the platelet count above a specific threshold.

TPO receptor agonists, romiplostim and eltrombopag, have been used in management of chronic thrombocytopenia due to ITP and liver disease and are being studied as a supportive medication in stem cell transplantation [86]. There is a case report in the pediatric transplant literature where romiplostim was used in the peri-transplant setting, which resulted in a platelet-transfusion-free liver transplant [87]. However at this time, there is no data to support use of TPO agonists outside of their approved indications following solid organ transplant.

\section{References}

1. Vanrenterghem Y, Ponticelli C, Morales JM, et al. Prevalence and management of anemia in renal transplant recipients: a European survey. Am J Transplant. 2003;3:835-45.

2. Yorgin PD, Scandling JD, Belson A, Sanchez J, Alexander SR, Andreoni KA. Late post-transplant anemia in adult renal transplant recipients. Am J Transplant. 2002;2:429-35.

3. Kolonko A, Pinocy-Mandok J, Kocierz M, et al. Anemia and erythrocytosis after kidney transplantation: a 5 year graft function and survival analysis. Transplant Proc. 2009;8:3046-51.

4. Przybylowski P, Malyszko J, Malyszko J. Anemia is a predictor of outcome in heart transplant recipients. Transplant Proc. 2009;41:322-3231.

5. Taegtmeyer AB, Rogers P, Breen JB, Barton PJ, Banner NR, Yacoub MH. The effects of pre- and post-transplant anemia on 1-year survival after cardiac transplantation. J Heart Lung Transplant. 2008;27:394-9.
6. Yazer $\mathrm{MH}$, Triulzi DJ. Immune hemolysis following ABOmismatched stem cell or solid organ transplantation. Curr Opin Hematol. 2007;14:664-70.

7. Audet M, Panaro F, Piardi T, et al. Passenger lymphocyte syndrome and liver transplantation. Clin Dev Immunol. 2009;2008:715769.

8. Shortt J, Westall GP, Roxby D. A 'dangerous' group O donor: severe hemolysis in all recipients of organs from a donor with multiple red cell alloantibodies. Am J Transplant. 2008;8:711-4.

9. Seltsam A, Hell A, Heymann G, Salama A. Donor derived alloantibodies and passenger lymphocyte syndrome in two of four patients who received different organs from the same donor. Transfusion. 2001:41:365-70.

10. Salerno CT, Burdine J, Perry EH, Kshettry VR, Hertz MI, Bolman IIIR. Donor-derived antibodies and hemolysis after ABOcompatible but nonidentical heart-lung and lung transplantation. Transplantation. 1998;65:261-4.

11. Panaro F, DeChristopher PJ, Rondelli D. Severe hemolytic anemia due to passenger lymphocytes after living related bowel transplant. Clin Transpl. 2004;18:332-5.

12. Winkelmayer WC, Kewalramani R, Rutstein M, Gabardi S, Vonvisger T, Chandraker A. Pharmacoepidemiology of anemia in kidney transplant recipients. J Am Soc Nephrol. 2004;15:1347-52.

13. Imoagene-Oyedeji AE, Rosas SE, Doyle AM, Goral S, Bloom RD. Posttransplantation anemia at 12 months in kidney recipients treated with mycophenolate mofetil: risk factors and implications for mortality. J Am Soc Nephrol. 2006;17:3240-7.

14. Augustine JJ, Knauss TC, Schulak JA, Bodziak KA, Siegel C, Hricik DE. Comparative effects of sirolimus and mycophenolate mofetil on erythropoiesis in kidney transplant patients. Am J Transplant. 2004;4:2001-6.

15. Pratap B, Abraham G, Srinivas CN, Bhaskar S. Post-renal transplant hemolytic uremic syndrome following combination therapy with tacrolimus and everolimus. Saudi J Kidney Dis Transpl. 2007;18:609-12.

16. Go O, Naqvi A, Tan A, Amzuta I, Lenox R. The spectrum of thrombotic thrombocytopenic purpura: a clinicopathologic demonstration of tacrolimus-induced thrombotic thrombocytopenic purpura in a lung transplant patient. South Med J. 2008;101:744-7.

17. Saito M, Satoh S, Kagaya H, et al. Thrombotic microangiopathy developing in early stage after renal transplantation with a high trough level of tacrolimus. Clin Exp Nephrol. 2008;12:312-5.

18. Maheshwari A, Mishra R, Thuluvath PJ. Post-liver transplant anemia: etiology and management. Liver Transpl. 2004;10:165-73.

19. Nosari A, Marbello L, De Carlis LG, et al. Bone marrow hypoplasia complicating tacrolimus (FK506) therapy. Int J Hematol. 2004;79:130-2.

20. Engelen W, Verpooten GA, Van der Planken M, Helbert MF, Bosmans JL, De Broe ME. Four cases of red blood cell aplasia in association with the use of mycophenolate mofetil in renal transplant patients. Clin Nephrol. 2003;60:119-24.

21. Naik PM, Lyon GM 3rd, Ramirez A, et al. Dapsone-induced hemolytic anemia in lung allograft recipients. J Heart Lung Transplant. 2008;27:1198-202.

22. Ash-Bernal R, Wise R, Wright SM. Acquired methemoglobinemia: a retrospective series of 138 cases at 2 teaching hospitals. Medicine. 2004;83:265-73.

23. Dunford LM, Roy DM, Hahn TE, et al. Dapsone-induced methemoglobinemia after hematopoietic stem cell transplantation. Biol Blood Marrow Transplant. 2006;12:241-2.

24. Balbi E, Leal CR, Pacheco-Moreira LF, et al. Treatment for recurrent hepatitis $\mathrm{C}$ virus infection after liver transplantation. Transplant Proc. 2009;41:891-4.

25. Glanville AR, Scott AI, Morton JM, et al. Intravenous ribavirin is a safe and cost-effective treatment for respiratory syncytial virus infection after lung transplantation. J Heart Lung Transplant. $2005 ; 24: 2114-9$. 
26. Ar MC, Ozbalak M, Tuzuner N, et al. Severe bone marrow failure due to valganciclovir overdose after renal transplantation from cadaveric donors: four consecutive cases. Transplant Proc. 2009;41:1648-53.

27. Elimelakh M, Dayton V, Park KS, et al. Red cell aplasia and autoimmune hemolytic anemia following immunosuppression with alemtuzumab, mycophenolate, and daclizumab pancreas transplant recipients. Haematologica. 2007;92:1029-36.

28. Mix TC, Kazmi W, Khan S, et al. Anemia: a continuing problem following kidney transplantation. Am J Transplant. 2003;3:1426-33.

29. Smith EP. Hematologic disorders after solid organ transplantation. Am Soc Hematol Am Soc Hematol Educ Program. 2010;2010:281-6.

30. Park JB, Kim DJ, Woo SY, et al. Clinical implications of quantitative real time-polymerase chain reaction of parvovirus B19 in kidney transplant recipients - a prospective study. Transpl Int. 2009;22:455-62.

31. Florea AV, Ionescu DN, Melhem MF. Parvovirus B19 infection in the immunocompromised host. Arch Pathol Lab Med. 2007;131:799-804.

32. Shekar K, Hopkins PM, Kermeen FD, Dunning JJ, McNeil KD. Unexplained chronic anemia and leukopenia in lung transplant recipients secondary to parvovirus B19 infection. J Heart Lung Transplant. 2008;27:808-11.

33. Marinella MA. Hematologic abnormalities following renal transplantation. Int Urol Nephrol. 2010;42:151-64.

34. Parker A, Bowles K, Bradley JA, et al. Management of posttransplant lymphoproliferative disorder in adult solid organ transplant recipients - BCSH and BTS guidelines. Br J Haematol. 2010;149:693-705.

35. Kato T, Yazawa K, Madono K, Saito J, Hosomi M, Itoh K. Acute graft-vs-host disease in kidney transplantation: case report and review of literature. Transplant Proc. 2009;41:3949-52.

36. Lo MM, Mo JQ, Dixon BP, Czech KA. Disseminated histoplasmosis associated with hemophagocytic lymphohistiocytosis in kidney transplant recipients. Am J Transplant. 2010;10:687-91.

37. Hughes WT, Armstrong D, Bodey GP, et al. Guidelines for the use of antimicrobial agents in neutropenic patients with cancer. Clin Infect Dis. 2002;34:730-51.

38. Hartmann EL, Gatesman M, Roskopf-Somerville J, Stratta R, Farney A, Sundberg A. Management of leukopenia in kidney and pancreas transplant recipients. Clin Transpl. 2008;22:822-8.

39. Dewit O, Moreels T, Baert F, et al. Limitations of extensive TPMT genotyping in the management of azathioprine-induced myelosuppression in IBD patients. Clin Biochem. 2011;44:1062-6.

40. Danesi R, Del Tacca M. Hematologic toxicity of immunosuppressive treatment. Transplant Proc. 2004;36:703-4.

41. The Tricontinental Mycophenolate Mofetil Renal Transplantation Study Group. A blinded, randomized clinical trial of mycophenolate mofetil for the prevention of acute rejection in cadaveric renal transplantation. Transplantation. 1996;61:1029-37.

42. Knoll GA, MacDonald I, Khan A, Van Walraven C. Mycophenolate mofetil dose reduction and the risk of acute rejection after renal transplantation. J Am Soc Nephrol. 2003;14:2381-6.

43. Fischer SA. Emerging viruses in transplantation: there is more to infection after transplant then CMV and EBV. Transplantation. 2008;86:1327-39.

44. Thomas LD, Hongo I, Bloch KC, Tang YW, Dummer S. Human ehrlichiosis in transplant patients. Am J Transplant. 2007;7: 1641-7.

45. Arakawa Y, Matsui A, Sasaki N, Nakayama T. Agranulocytosis and thrombocytopenic purpura following measles infection in a livingrelated orthotopic liver transplantation recipient. Acta Paediatr Jpn. 1997;39:226-9.

46. Wiwanitkit V. Dengue infection in kidney transplant patients: an appraisal on clinical manifestation. Iran J Kidney Dis. 2010;4:168.
47. Eid AJ, Brown RA, Patel R, Razonable RR. Parvovirus B19 infection after transplantation: a review of 98 cases. Clin Infect Dis. 2006;43:40-8.

48. Karakus S, Arin BE, Arat Z, Karakayali H, Haberal M. Impact of hepatitis serology on development of leukopenia after solid organ transplantation. Transplant Proc. 2008;40:199-201.

49. Razeghi E, Hadadi A, Mansor-Kiaei M, Molavi M, Khashayar P, Pourmand G. Clinical manifestation, laboratory findings, and the response of treatment in kidney transplant recipients with CMV infection. Transplant Proc. 2007;39:993-6.

50. Simmons P, Kaushansky K, Torok-Storb B. Mechanisms of cytomegalovirus-mediated myelosuppression: perturbation of stromal cell function versus direct infection of myeloid cells. Proc Natl Acad Sci U S A. 1990;87:1386-90.

51. Kute VB, Vanikar AV, Shah PR, et al. Post-renal transplant cytomegalovirus infection: study of risk factors. Transplant Proc. 2012;44:706-9.

52. Cooke ME, Potena L, Luikart H, Valantine HA. Peripheral blood leukocyte counts in cytomegalovirus infected heart transplant patients: impact of acute disease versus subclinical infection. Transplantation. 2006;82:1419-24.

53. Rane S, Nada R, Minz M, Sakhuja V, Joshi K. Spectrum of cytomegalovirus-induced renal pathology in renal allograft recipients. Transplant Proc. 2012;44:713-6.

54. De Keyzer K, Van Laecke S, Peeters P, Vanholder R. De novo thrombotic microangiopathy induced by cytomegalovirus infection leading to renal allograft loss. Am J Nephrol. 2010;32:491-6.

55. Zuber J, Le Quintrec M, Sberro-Soussan R, Loirat C, FremeauxBacchi V, Legendre C. New insights into postrenal transplant hemolytic uremic syndrome. Nat Rev Nephrol. 2011;7:23-35.

56. Ramasubbu K, Mullick T, Koo A, et al. Thrombotic microangiopathy and cytomegalovirus in liver transplant recipients: a case-based review. Transpl Infect Dis. 2003;5:98-103.

57. Hachem RR, Yusen RD, Chakinala MM, Aloush AA, Patterson GA, Trulock EP. Thrombotic microangiopathy after lung transplantation. Transplantation. 2006;81:57-63.

58. Fischer SA. Emerging viruses in transplantation: there is more to infection after transplant than CMV and EBV. Transplantation. 2008;86:1327-39.

59. Caires RA, Marques IDB, Repizo LP, et al. De novo thrombotic microangiopathy after kidney transplantation: clinical features, treatment, and long-term patient and graft survival. Transplant Proc. 2012;44:2388-90.

60. Fouad YM. Chronic hepatitis C-associated thrombocytopenia: aetiology and management. Trop Gastroenterol. 2013;34:58-67.

61. Smith EP. Hematologic disorders after solid organ transplantation. ASH Educ Program Book. 2010;2010:281-6.

62. Devadoss CW, Vijaya VM, Mahesh E, Venkataramana SR, Girish MS. Tacrolimus associated localized thrombotic microangiopathy developing in early stage after renal transplantation. J Clin Diagn Res. 2012;6:1786-8.

63. Boyer NL, Niven A, Edelman J. Tacrolimus-associated thrombotic microangiopathy in a lung transplant recipient. BMJ Case Rep. 2013;2013:bcr2012007351.

64. Nwaba A, MacQuillan G, Adams LA, et al. Tacrolimus-induced thrombotic microangiopathy in orthotopic liver transplant patients: case series of four patients. Intern Med J. 2013;43:328-33.

65. Fortin MC, Raymond MA, Madore F, et al. Increased risk of thrombotic microangiopathy in patients receiving a cyclosporin-sirolimus combination. Am J Transplant. 2004;4:946-52.

66. Minson Q, Gentry CA. Analysis of linezolid-associated hematologic toxicities in a large veterans affairs medical center. Pharmacotherapy. 2010;30:895-903.

67. Bernstein WB, Trotta RF, Rector JT, Tjaden JA, Barile AJ. Mechanisms for linezolid-induced anemia and thrombocytopenia. Ann Pharmacother. 2003;37:517-20. 
68. El-Khoury J, Fishman JA. Linezolid in the treatment of vancomycinresistant enterococcus faecium in solid organ transplant recipients: report of a multicenter compassionate-use trial. Transpl Infect Dis. 2003;5:121-5.

69. Radunz S, Juntermanns B, Kaiser GM, et al. Efficacy and safety of linezolid in liver transplant patients. Transpl Infect Dis. 2011;13:353-8.

70. Attassi K, Hershberger E, Alam R, Zervos MJ. Thrombocytopenia associated with linezolid therapy. Clin Infect Dis. 2002;34:695-8.

71. Lo GK, Juhl D, Warkentin TE, Sigouin CS, Eichler P, Greinacher A. Evaluation of pretest clinical score (4 T's) for the diagnosis of heparin-induced thrombocytopenia in two clinical settings. J Thromb Haemost. 2006;4:7.

72. Bachmann R, Bachmann J, Lange J, Nadalin S, Konigsrainer A, Ladurner R. Incidence of heparin-induced thrombocytopenia type II and postoperative recovery of platelet count in liver graft recipients: a retrospective cohort analysis. J Surg Res. 2014;186:429-35.

73. Kaneko J, Sugawara Y, Tamura S, Togashi J, Matsui Y, Makuuchi M. Heparin-induced thrombocytopenia after liver transplantation. Transplant Proc. 2008;40:1518-21.

74. Dracopoulos S, Vougas V, Kassimatis TI, Theodoridis T, Ali ME, Apostolou T. Heparin-induced thrombocytopenia type II: a serious hazard in preemptive renal transplantation: a case report. Transplant Proc. 2007;39:3481-4.

75. Muzaffar M, Li X, Ratnam S. Successful preemptive renal retransplantation in a patient with previous acute graft loss secondary to HIT type II: a case report and review of literature. Int Urol Nephrol. 2012;44:991-4.

76. Chaturvedi S, Kohli R, McCrae K. Over-testing for heparin induced thrombocytopenia in hospitalized patients. J Thromb Thrombolysis. 2015;40(1):12-6.

77. Lugao Rdos S, Motta MP, de Azevedo MF, et al. Immune thrombocytopenic purpura induced by intestinal tuberculosis in a liver transplant recipient. World J Gastroenterol. 2014;20:8304-8.
78. Einollahi B. Renal transplantation and idiopathic thrombocytopenic purpura: two case reports. Transplant Proc. 2009;41:2923.

79. Taylor RM, Bockenstedt P, Su GL, Marrero JA, Pellitier SM, Fontana RJ. Immune thrombocytopenic Purpura following liver transplantation: a case series and review of the literature. Liver Tranplant. 2006;12:11.

80. Pereboom IT, de Boer MT, Haagsma EB, van der Heide F, Porcelijn L, Lisman T, Porte RJ. Transmission of idiopathic thrombocytopenic purpura during orthotopic liver transplantation. Transplant Int. 2010;23:3.

81. Kim KJ, Baek IW, Yoon CH, Kim WU, Cho CS. Thrombotic risk in patients with immune thrombocytopenia and its association with antiphospholipid antibodies. Br J Haematol. 2013;161: 706-14.

82. Yang YJ, Yun GW, Song IC, et al. Clinical implications of elevated antiphospholipid antibodies in adult patients with primary immune thrombocytopenia. Korean J Intern Med. 2011;26:449-54.

83. Chatzipetrou MA, Tsaroucha AK, Weppler D, et al. Thrombocytopenia after liver transplantation. Transplantation. 1999;67:702-6.

84. Plevak DJ, Halma GA, Forstrom LA, et al. Thrombocytopenia after liver transplantation. Transplant Proc. 1988;20:630-3.

85. Pham PT, Danovitch GM, Wilkinson AH, et al. Inhibitors of ADAMTS13: a potential factor in the cause of thrombotic microangiopathy in a renal allograft recipient. Transplantation. 2002;74:1077-80.

86. Liesveld JL, Phillips GL 2nd, Becker M, et al. A phase 1 trial of eltrombopag in patients undergoing stem cell transplantation after total body irradiation. Biol Blood Marrow Transplant. 2013;19:1745-52.

87. Minowa K, Arai K, Kasahara M, et al. Romiplostim treatment allows for platelet transfusion-free liver transplantation in pediatric thrombocytopenic patient with primary sclerosing cholangitis. Pediatr Transplant. 2014;18:E212-5. 